\title{
La intervención pedagógica en entornos rurales: evaluación de programas de formación
}

\author{
Rosa María Pascual Matesanz \\ Colegio Rural Agrupado "Ana de Austria"
}

\begin{abstract}
Resumen
Las iniciativas integradas de la Unión Europea denominadas LEADER, dirigidas al desarrollo de sus zonas desfavorecidas, incluyen la formación de los Recursos Humanos. Este artículo se centra en la evaluación de las acciones formativas en LEADER I y II llevadas a cabo en la comarca castellano-leonesa Pinares-El Valle (España). Con esta finalidad se ha adoptado el modelo CIPP de Stufflebean. Se tratan estadísticamente los datos, obtenidos mediante cuestionarios y entrevistas a los participantes, en los niveles descriptivo, bivariable y multivariable; tomamos los criterios adaptación, eficacia e impacto en las personas y la zona. Los resultados obtenidos indican la bondad de las acciones formativas. Proponemos su continuación.
\end{abstract}

Palabras Clave: Recursos Humanos, Formación, Evaluación, Adaptación, Eficacia, Impacto.

\section{Summary}

The integral initiatives of European Union designated $L E A D E R$, directed to the development of its depressed zones, include the formation of human resources. We evaluate the formative actions inside LEADER I and II realized in the Pinares-El Valle county, placed into Castilla and León (Spain). We adopt the Stufflebeam's CIPP evaluative model with its variables context, input, process and product.The information were obtained through questionnaires and interviews answered by the participants. We processed the data statistically in the levels descriptive, bivariable and multivariable, with the views: adaptation, efficacy and impact about the persons and the zone. The results obtained indicate the goodness of the formatives actions. We propose its continuation.

KeY words: Human Resourses, Formation, Evaluation, Adaptation, Efficacy, Impact.

La intervención pedagógica en las zonas rurales se caracteriza, en muchos casos, por la falta de un programa sistemático para realizar tal intervención que sea fundamento y guía de la acción educativa. Nuestro principal propósito en este trabajo es poner de manifiesto la necesidad de intervenir en los contextos rurales con rigor científico, donde la evalua ción desempeñe un papel principal orientado a la toma de decisiones sobre su posible efectividad. 
La evaluación que proponemos no se centra únicamente en la finalización del programa, es más, se extiende a todas las fases, desde el diseño y selección de las medidas de evaluación que se emplearán, hasta la divulgación de los conocimientos adquiridos, mediante dicha evaluación, a otras zonas de similares características. Partiendo de este planteamiento y de la realidad de la intervención educativa en el entorno rural, cree mos conveniente la necesidad de planificar y e valuar un programa de desarrollo de la Unión Europea destinado al mundo rural: el Programa LEADER.

Esta institución internacional, principalmente en las últimas décadas, está poniendo el mayor empeño en la atención a sus zonas más desfavorecidas, como son las zonas rurales, con grandes carencias en los ámbitos socioeconómico y cultural, en la mayoría de las ocasiones marginadas y deprimidas. Estos entornos se caracterizan por una gran dependencia de la agricultura, una escasa formación de la población y un tejido social débil. En estas circunstancias han influido diversos factores como el aislamiento geográfico, las deficientes vías de comunicación y los difíciles accesos a las zonas industriales y a los centros de cultura. Las zonas rurales ocupan más del 80\% del territorio de la Unión, por lo que resultan muy necesarias las iniciativas destinadas a posibilitar la mejora de las condiciones de vida de sus habitantes y del lugar en el que se desenvuelve su vida.

La Comisión Europea, en su intento de afrontar y resolver estas carencias, ha proyectado el establecimiento de las directrices para subvenciones globales integradas y ha invitado a los Estados miembros a presentar propuestas, en el marco de una Iniciativa Comunitaria de Desarrollo Rural: LEADER “Liasson Entre Activités du Dèveloppement de l'Économie Rural” (Relaciones entre Actividades de Desarrollo de la Economía Rural). La Iniciativa se inscribe entre las concebidas por la Comisión para apoyar actuaciones que contribuyan a resolver problemas de una dimensión europea especial. Presenta las siguientes características: $A p o$ yan las operaciones de cooperación transnacional, transfronteriza e interregional y se llevan a cabo por un método ascendente "de abajo a arriba", es decir, se programan y realizan atendiendo a las necesidades detectadas de los que se acogen a ellas, revirtiendo más tarde en el desarrollo y progreso de los pueblos que forman la Comunidad. Además, especialmente el programa LEADER se propone:

a) Potenciar la participación de las poblaciones regionales y locales en sus organizaciones;

b) Complementar las inversiones estatales en los diversos sectores de producción;

c) Fomentar la organización en cooperativas; d) Propiciar la formación y creación de empleos y capacidades.

La Iniciativa LEADER comenzó en 1991 con LEADER I (Comunicación de la Comisión a los Estados miembros 91/C 31/14, DOC 73 de 19-3). Continuó con LEADER II (Comunicación de la Comisión a los estados miembros 94/C 180/12, DOCE 180 del 1-7-1994). Actualmente, se está llevando a cabo el LEADER PLUS (Decisión de la Comisión de las Comunidades Europeas n ${ }^{\circ}$ C, 2001, 2176 de 20 de agosto).

Estos Programas se están aplicando en todos los países que forman la Unión Europea, en número diferente, atendiendo a las regiones de los mismos consideradas "de Objetivo 1". Se tiene en cuenta la renta per cápita, que no debe exceder el $75 \%$ de la media de los países de la Unión. Entre las subvenciones integradas para llevar a cabo el desarrollo de diversas "Medidas" o "Pa rtes", de las que se componen los Programas LEADER, ocupa un lugar preferente la formación. 


\section{Importancia de la formación de los Recursos Humanos en el desarrollo de los pueblos}

La formación de los Recursos Humanos es uno de los temas que va tomando cada vez mayor relevancia en diversos campos, especialmente en el de la educación. Hoy día, constituye un elemento de preocupación a escala mundial y uno de los aspectos más importantes al que se dirige una amplia gama de instituciones dedicadas al desarrollo de los pueblos. Se habla mucho de capital humano, concepto que ha superado concepciones materialistas, considerándose como capital no sólo las formas visibles y apreciables por los sentidos, como los productos naturales, sino también los aspectos como las capacidades, heredadas o adquiridas. Esta noción resulta útil para el análisis de las diferentes actividades organizadas que aumentan aquellas capacidades humanas que mejoran la perspectiva de poder alcanzar mejores rentas. Así, la inversión en educación proporciona beneficios sociales y económicos al hacer posible el desarrollo de conocimientos y capacidades. Para que sea efectiva tal inversión, según afirmaciones de la OCDE debe afectar a todas las etapas de la educación y de la formación humana y promover la adquisición de conocimientos a lo largo de la vida. Por tanto, se concede un lugar destacado al capital humano, llamado también capital inmaterial o capital intangible. Estas expre siones incluyen, en primer lugar, la educación, además de la aptitud para la innovación.

La Unión Europea, desde sus comienzos, ha ido incluyendo e incrementando de manera paulatina la atención a la formación, tanto en sus Tratados constitutivos como en sus distintos foros. En el planteamiento de su política educativa, la formación de los recursos humanos se intensifica después de Maastricht, (1992), al hacerse explícita en el propio Tratado. Posteriormente, la Comisión publica también diversos textos claves entre los que destacan la declaración de 1996 como el Año Europeo de la Educación y de la Formación Permanente. Por otra parte, los Consejos Europeos celebrados en los que tratan sobre el tema, culminan con el de Lisboa (2000). En el mismo, se elaboró un Memo randum sobre el aprendizaje permanente para posibilitar en Europa una estrategia global, basada en la importancia otorgada a los aprendizajes formales, no formales e info rmales. Posteriormente, esta misma temática está presente en prácticamente todos los Consejos como el de Estocolmo (2001) y, en concreto, en el de Laeken (2001), donde se propone que los países europeos logren, antes del 2010, "la economía basada en el conocimiento más dinámica del mundo".

\section{2. Necesidad de evaluar}

Se hace necesario conocer si estos programas deben continuar o, si de hacerlo, habría que modificar algún aspecto. Hay que tener en cuenta, además, la necesidad de evaluar, ante los retos que continuamente debe afrontar una sociedad en desarrollo para alcanzar las metas propuestas y para que la aplicación de estos Programas de formación, tan peculiares, puedan alcanzar su objetivo: contribuir al enriquecimiento de la persona, a su formación personal y profesional, y a que la zona recobre vitalidad y adquiera un mayor desarrollo.

En esta investigación nos centramos en uno de los citados Programas LEADER, el denominado LEADER URBIÓN, que desarrolla sus acciones en la zona de Pinares-El Valle, ubicada en las provincias de Soria y de Burgos, de la Comunidad Autónoma de Castilla y León. Nuestra aportación consistirá en la evaluación de la formación, es decir, de las acciones formativas llevadas a cabo en el desarrollo de las "Medidas" o "Partes" dirigidas a esta cuestión. Para ello, se contará con la opinión de los participantes o protagonistas principales -técnicos en formación, alumnos y líderes comunitarios- a fin de hacer rea- 
lidad la "participación de la gente", idea-eje que impregna estos Programas.

\section{3. Planteamiento del problema. Objetivos}

Tras las consideraciones anteriores planteamos el problema objeto de evaluación. Así podemos preguntarnos: ¿El Programa LEADER es adecuado para lograr la formación de los recursos humanos? En otras palabras: ¿Son eficaces las acciones formativas llevadas a cabo en el Programa LEADER URBIÓN, para contribuir al progreso de las personas y de la zona donde se aplica?

Una vez formulado el interrogante, procedemos a la elaboración de un modelo para evaluar la incidencia de las"Medidas" dedicadas a la formación (acciones formativas singulares) del Programa mencionado, tanto en las personas como en la zona. De aquí se deriva nuestro objetivo general: Evaluar la acción formativa de los Programas LEADER I y LEADER II de la Unión Europea, es decir, la realización de sus Cursos y Jornadas técnicas para conseguir una formación específica de los recursos humanos de la zona. Como objetivos específicos figuran los siguientes:

a) Analizar el grado de adaptación del Programa de formación a las características personales de los alumnos, a sus intereses y a las necesidades de la zona.

b) Determinar la eficacia del Programa de formación. Relación objetivos-logros personales.

c) Comprobar el impacto producido en la zona por la aplicación del Programa de formación. Relación situación de referencialogros-penetración social.

\section{Metodología}

La evaluación se planificó y se llevó a cabo en dos fases:

1) Preparación: En esta etapa iniciamos el análisis sistemático y reflexivo de la literatura relativa a la formación de los recursos humanos, en general y de los Programas de desarrollo sobre el campo que nos ocupa, con autores como Ander Egg (2000). Cernea (1995). Marchioni (2001). Valcárcel (1987) y Vachon (2001). Además, se realizó un estudio sistemático de los documentos de la Unión Europea respecto al estado de sus diversas regiones y a sus políticas en materia de desarrollo, (Directivas, Comunicaciones, etc.), más la legislación actual española relativa a este tema. Por otra parte, revisamos los estudios y modelos evaluativos existentes, así como las evaluaciones sobre problemáticas similares. Esta parte hace referencia al marco teórico consulta obligada para emprender a continuación el trabajo empírico.

2) Elaboración y aplicación de un diseño de evaluación específico para la realidad estudiada. Nuestra propuesta de evaluación tiene como punto de partida las interrelaciones que presentan los elementos implicados en la Forma ción de los recursos humanos, aspecto relevante de los programas LEADER, con su indispensable evaluación. Al ser el principal objetivo de dicho Programa lograr el desarrollo de la comunidad, consideramos necesaria una visión global de todos los elementos que interactúan en la comunidad. Sus particulares estructura y características se traducen en unos problemas y unas necesidades concretas, de los que deben tomar conciencia las autoridades correspondientes a fin de proporcionar las medidas oportunas, entre las que figuran las relativas a la formación. Los resultados de dicha evaluación vuelven a la comunidad para mejorarla.

\subsection{Modelo de evaluación. Desarrollo}

Este trabajo se encuentra en la línea de la investigación evaluativa. Se trata de una investigación aplicada. La metodología es de carácter descriptivo y empírico, teniendo en cuenta tanto la dimensión cualitativa como cuantitativa. Referente al modelo de evaluación

[ 108 ] SIPS - PEDAGOGÍA SOCIAL (ISSN-1139-1723) № 14 TERCERA ÉPOCA 
más conveniente para utilizar, con las debidas adaptaciones a la situación particular que nos ocupa, se seleccionó el Modelo CIPP de Stufflebeam, que comprende el estudio del Contexto, Entrada (Input), Proceso y Producto.

u Evaluación del contexto: se centra en la zona donde se aplica el Programa: se trata de determinar los rasgos de la misma, así como de su población.

u Evaluación de entrada: la componen variables relativas al contenido del programa de formación, la oportunidad del mismo, las necesidades de la población, las estrategias empleadas para llevar a cabo la acción formativa, la oferta educativa, la demanda de formación, las características de los técnicos de formación y de los participantes.

u Evaluación del proceso: se trata de la puesta en práctica del programa en aspectos tales como: su organización, los problemas surgidos, las modificaciones, etc.

Como criterio principal de evaluación a aplicar en estas tres dimensiones se ha utilizado el de: adaptación del programa de formación a los componentes de los tres aspectos citados. Como ya se ha señalado, se ha utilizado la descripción como metodología y ha primado la informa ción cualitativa. Como base documental se ha tomado, por una parte, la documentación existente en la entidad promotora y coordinadora: Asociación Pinares-El Valle-ASOPIVA y, por otra, los materiales de los cursos (trípticos, folletos y material didáctico, productos generados...), así como la literatura existente sobre los Programas LEADER.

u Evaluación del producto: se centra en los efectos y logros obtenidos derivados de la aplicación del programa, tanto para las personas participantes como para la zona. Se ha tomado como criterios de evaluación la adaptación, la eficacia y el impacto.

Para la recogida de información, hemos elaborado cuestionarios y entrevistas específic a s. Los primeros se aplicaron tanto a los técnicos en formación como a los alumnos participantes. Las segundas, para recoger opiniones y valoraciones de los líderes comunitarios (alcaldes, organizadores y gestores del programa) y de los habitantes de la zona.

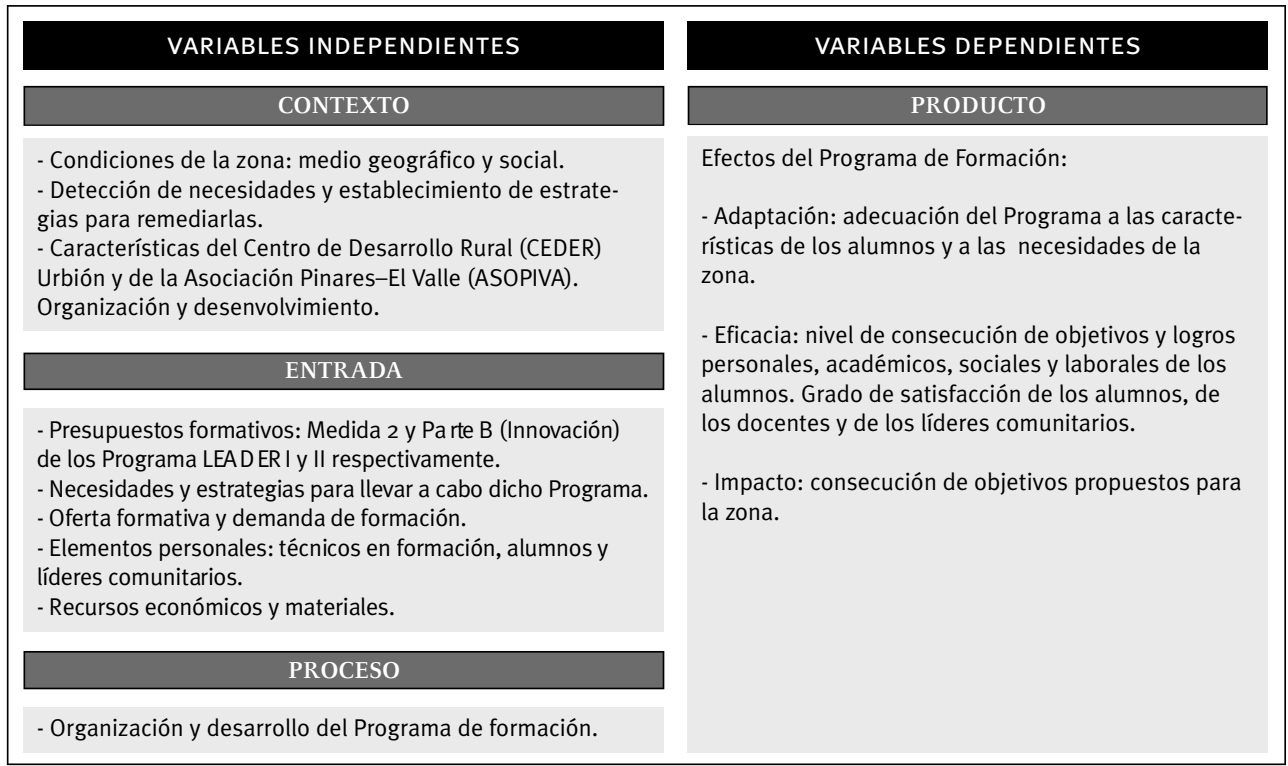




\subsection{Instrumentos de recogida de datos}

Los cuestionarios se estructuraron en bloques que reflejan y agrupan todos los indicadores de las variables citadas. Para tratar de conseguir fiabilidad y validez exigida en este tipo de trabajos nos preocupó cumplir las condiciones de: valor de verdad, aplicabilidad, consistencia y neutralidad, siguiendo los criterios de autores como Colas y Pérez Serrano (1994: 275). Con este propósito, en primer lugar se hicieron validar por 26 jueces, todos ellos expertos en este campo como docentes universitarios de educación, de sociología y de geografía, así como educadores de personas adultas. El 95\% de las cuestiones resultaron valoradas en las puntuaciones más altas (muy adecuada-bastante adecuada) . En cuanto a la fiabilidad utilizamos el coeficiente ALFA de Cronbach, método denominado de consistencia interna. Resultó suficiente el coeficiente obtenido, dadas las características de los datos (Fox, 1987).

Además, se realizaron procesos de triangulación que avalan diversos autores (Pérez Serrano, 1994) dado que, en ciencias humanas dichos procesos explican de manera más concreta la riqueza y la complejidad del comportamiento humano, al estudiarlo desde diferentes perspectivas, utilizando datos tanto cuantitativos como cualitativos. Siguiendo las recomendaciones de distintos expertos en la materia los instrumentos se aplicaron a toda la población objeto de estudio.

\section{Análisis e interpretación de los resultados}

El análisis y el tratamiento de la información se ha realizado con la ayuda del paquete de programas estadísticos: el Statistical Package for Social Sciences (SPSS). Los datos se han tratado en tres niveles:

Primer nivel descriptivo, para determinar las propiedades más relevantes que sirven de partida para análisis posteriores.

Segundo nivel bivariable. La estadística inferencial la utilizamos para determinar qué diferencias de las halladas se deben al azar y cuáles no (fijamos un nivel de confianza del 95\% para las afirmaciones que se realizan). El estadístico utilizado es el Análisis de Varianza de las variables, cruzando las más interesantes. Las pruebas aplicadas han sido la "U" de Mann Withney y el "Análisis de Variabilidad" de Kruskal Wallis. Los estudios posteriores de significatividad se han realizado siguiendo el procedimiento de las "dife rencias mínimas significativas".

Tercer nivel multivariable. Hemos aplicado el Análisis Factorial, con la técnica de las componentes principales, que permite aglutinar, desde la matriz de correlaciones, las componentes o factores que explican la varianza total (ocho factores rotados con el método Varimax, para facilitar su interpretación).

\subsection{Análisis del contexto}

La zona en que se ha realizado la investigación comienza, desde los años 60 del pasado siglo, a despoblarse a causa de la emigración. El crecimiento se detiene sobre todo en los pequeños núcleos de población donde ésta envejece. Este fenómeno es más leve en los núcleos mayores por concentrarse allí algún servicio o absorber alguno de los anteriores como el centro educativo o el centro de salud. $\mathrm{H}$ ay que anotar que, a medida que el sector industrial ha ido avanzando, se ha ido configurando la marginación de las zonas rurales. Las profesiones tradicionales necesitan adaptarse a los nuevos procesos de producción, tecnología y mecanización para que resulten rentables. Sus rasgos más relevantes son:

u Población: la densidad de población es de 11 hab/Km. cuadrado. Más de la mitad de los municipios tienen menos de 500 habitantes, con tendencia a descender y no tienen centro educativo. Un $6 \%$ de las poblaciones tienen tendencia ascendente que coincide con

[ 110 ] SIPS - PEDAGOGÍA SOCIAL (ISSN-1139-1723) № 14 TERCERAÉPOCA 
la existencia de alguna industria. La población activa es de 6.188 hab. (sector primario $38 \%$, secundario $30 \%$ y terciario $32 \%$ )

u Comunicaciones: son escasas y, a veces, difíciles. El entramado social es débil.

u Acciones llevadas a cabo: a finales de los años 80 del pasado siglo, los alcaldes decidieron movilizarse con el objetivo de conseguir el desarrollo de la zona, remediando las carencias y acrecentando los recursos. De los estudios realizados se desprende la siguiente información:

\section{CARENCIAS}

Despoblación

Escasez de industria

Falta de formación personal y profesional

Falta de información para acometer el desarrollo de lazona

\section{POTENCIALIDADES}

Silvicultura

Riqueza paisajística

Turismo

Fruto de esta iniciativa y de la toma de conciencia correspondiente se constituye el Grupo de Acción Local de 32 ayuntamientos que da lugar a la asociación ASOPIVA. Dicha entidad persigue "el desarrollo integral a través de la gestión e incentivación de iniciativas de desarrollo rural".

\subsection{Análisis de entrada}

Se exponen, seguidamente, los elementos más sobresalientes que se desprenden de esta fase de la investigación:

u Presupuestos formativos: el objetivo principal radica en conseguir el desarrollo de esta zona rural, lo que requiere la puesta en práctica de "programas integrados" en los que la formación constituye una de sus "Medidas" o "Partes" (Medida 2 y Parte B) LEADER I y II u Necesidades de formación y estrategias para llevar a cabo el Programa: los líderes comunitarios, tras la elaboración de los análisis apropiados, han establecido unas estrategias de actuación para ajustar la oferta a la demanda, al apreciarse la necesidad de formación personal y profesional adaptada a las necesidades de estas personas y al lugar donde se desenvuelve su vida.

u Cauces de formación en LEADER I y II. Se recogen en el siguiente cuadro:

\begin{tabular}{|l|l|}
\hline \multicolumn{2}{|c|}{$\begin{array}{l}\text { ACCIONES FORMATIVAS } \\
\text { Cursos y Jornadas Técnicas }\end{array}$} \\
\hline $\begin{array}{l}\text { 1. Recursos endógenos } \\
\text { - agricultura: } 35 \%\end{array}$ & $\begin{array}{l}\text { 2. Diversificación } \\
\text { - ganadería: } 15 \%\end{array}$ \\
$\begin{array}{l}\text { - pymes: } 30 \% \\
\text { que: } 50 \% \text { - medio ambiente y turis- }\end{array}$ & $\begin{array}{l}\text { mo: } 20 \% \\
\text { - aplicaciones o derivados } \\
\text { del bosque, agricultura y } \\
\text { ganadería: } 30 \% \\
\text { - organización de la socie- } \\
\text { dad rural: } 15 \%\end{array}$ \\
\hline
\end{tabular}

u Modalidad educativa: educación no formal. Se considera un sector educativo heterogéneo, disperso y amplio, puesto que se trata de crear, paralelamente a la escuela, otros medios y entornos educativos complementarios para atender a otras demandas emergentes. Numerosos autores han destacado su pertinencia e idoneidad para acciones educativas de este tipo, especialmente por los responsables europeos en este campo.

Centrándolos en los elementos personales, los rasgos que configuran el perfil de los técnicos en formación es el siguiente: predominan los hombres (80\%); de mediana edad (30-49 años); poseen alta formación académica $(70 \%$ son licenciados) y la profesión predominante es la de ingenieros de montes. En cuanto a los alumnos cabe indicar que su perfil es muy variado. Sí se produce un predominio de los 
hombres (3 a 2), pero el sector de edad comprende entre los 16 y 70 años. En cuanto a su formación académica, casi la mitad posee el título de Bachillerato, algo más de la cuarta parte son diplomados o licenciados universitarios y el resto tienen estudios primarios. En relación a su profesión, los más jóvenes, recién terminados sus estudios básicos, se encuentran a la búsqueda del primer empleo. Son escasos los estudiantes universitarios y bastante más numerosos los gana deros, agricultores, y amas de casa. Atendiendo al aspecto laboral, predominan los trabajadores por cuenta ajena (50\%); los autónomos son un $35 \%$ y no tienen trabajo el 18\%. Del grupo de los líderes comunitarios cabe afirmar que son personas cualificadas, dado que el $80 \%$ posee estudios medios e incluso superiores. Por su parte, los alcaldes tienen una calificación académica muy variada.

\subsection{Análisis del proceso}

Las variables que se han contemplado son: organización, desarrollo, participación y proceso metodológico-didáctico. Desde una perspectiva global, hay que señalar que la valoración es positiva. Los líderes comunitarios realizaron reuniones previas para detectar las carencias y potencialidades de la zona. Para determinar las necesidades y las demandas de formación, recabaron la ayuda de expertos en la materia de los cursos (ingenieros agrónomos, ingenieros forestales, veterinarios, etc.) pertenecientes a las escuelas Técnicas y Universidades de Castilla y León. También, consultaron experiencias extranjeras para la determinación de contenidos teóricos, prácticos, la metodología y la elaboración de los materiales. Este proceso sirvió y fue la base para elaborar la programación y determinar el lugar más conveniente para su puesta en marcha. Realizaron, asimismo, una campaña de difusión para asegurarse de que la información llegaba a todos los posibles interesados.
Se estableció un sistema de seguimiento y supervisión, que facilitó modificar aquellos aspectos o elementos que no daban el resultado esperado o bien los que más se demandaban. Al finalizar, el 85\% de los participantes estaba satisfecho mientras que el resto cambiaría, en cierta medida, lo referente a la preparación de los medios y materiales, así como el lugar de celebración. Junto a lo anterior, se detectaron nuevas demandas formativas para el futuro, especialmente, en: tecnología de la información, turismo y hostelería.

Se valoró positivamente la metodología didáctica empleada, así como la supervisión del desarrollo de todas las acciones formati vas. Los participantes mostraron un alto interés, siendo el abandono muy escaso pues, en el conjunto de cursos y jornadas, nunca llegó a superar el $2 \%$ de media.

Todas las personas implicadas: organizadores, técnicos en formación y alumnos se mostraron de acuerdo en que para conseguir el progreso de estas zonas debe prestarse, por parte de la Administración competente, especial atención a la formación, como elemento principal para conseguir el desarrollo de sus gentes.

\subsection{Análisis del producto}

A partir de los datos obtenidos en la investigación (Pascual, R., 2005) presentamos la siguiente síntesis de los aspectos de mayor relevancia:

a) Adaptación: con relación a los técnicos de formación se puede afirmar que se ha podido disponer de verdaderos profesionales con una formación docente adecuada, como lo demuestra el equilibrio conseguido en las actuaciones teórico-prácticas, por la atención prestada a la preparación de todos los aspectos de los cursos y por la alta valoración otorgada por los alumnos. Los alumnos participantes, por su parte, opinan que resulta muy interesante el empleo de una metodología 
diversa y participativa. Además, valoran muy pertinentes y eficaces los métodos utilizados, la adecuación de los horarios y la diversidad temática. Por su parte, los líderes comunitarios aseguran que su principal preocupación consiste en la ampliación de la oferta para mejorar la adaptación a los intereses de los participantes, en cuanto a temas, lugar de celebración, prácticas y material. De igual modo, se ha constatado el interés de los técnicos en formación, alumnos y líderes comunitarios por la formación, que consideran, en este caso, específica y adaptada a las carencias de la comarca, al tiempo que constituye un elemento primordial para su desarrollo.

Se puede concluir que el Programa se ha adaptado en un alto grado a las características personales, académicas y sociales de los alumnos. A pesar de la gran variedad de intereses de los usuarios, tanto éstos como los docentes aseguran que las distintas acciones educativas les han resultado muy satisfactorias. Las escasas discrepancias detectadas pueden considerarse dentro de lo esperable, si bien las reivindicaciones expresadas deben tenerse en cuenta en el futuro.

b) Eficacia: sobre su participación y satisfacción los técnicos en formación opinan, en un porcentaje superior al 95\%, que los cursos han contribuido a su enriquecimiento personal y profesional, así como al fomento de las relaciones humanas. Similar índice se obtiene entre el colectivo de los alumnos sobre variables como motivación, interés y participación A su juicio, las principales causas que han incidido en dicha participación radican en que los cursos han contribuido a: adquirir conocimientos y ampliarlos -siendo los que más se inclinan por esta respuesta los estudiantes y los de menor edad-, mejorar sus expectativas de empleo y favorecer las relaciones sociales.

Sobre la importancia y utilidad de estas acciones formativas, los mencionados participantes destacan como rasgos más sobresalientes el haberles proporcionado la oportu- nidad de obtener un diploma (en especial los estudiantes y los ganaderos) y el hecho de que responden a las necesidades de la zona (91\%). Asimismo, subrayan que es preciso ampliar la oferta con cursos sobre hostelería, ganadería e informática (el 75\%), tanto en beneficio propio como del territorio.

La información recogida sobre su satisfacción y logros alcanzados, revela aspectos positivos como: considerar adecuada la formación específica de cada curso $(73 \%)$ y el que les haya sido de gran utilidad para adquirir y ampliar el nivel de conocimientos, mejorando sus expectativas a la vez que desarrollar mejor su profesión (72\%). Por otra parte, se obtiene valores altos (entre el 60\% y $77 \%$ ) al asegurar que se han desarrollado actitudes sociales positivas, tales como: compañerismo, intercambio de experiencias, solidaridad y cooperación. Resulta significativo que las amas de casa sean las más favorables a esta estimación.

Los líderes comunitarios, alcaldes, organizadores y gestores, en algo más del 75\%, sostienen que las actividades formativas han suscitado gran interés y que la participación se ha ido incrementando a lo largo de la experiencia $(78 \%)$. Del mismo modo, mantienen que las actuaciones educativas han contribuido de forma decisiva: a la formación para el ejercicio profesional desempeñado; a mejorar las expectativas personales; a fomentar las relaciones entre las personas; a elevar el nivel cultural y a consolidar, conservar o crear el puesto de trabajo. Además, su valoración es unánime al considerar que las temáticas seleccionadas han resultado de interés para las personas y para la zona. Piensan que un factor decisivo para lograr los objetivos propuestos ha sido la colaboración establecida entre la entidad responsable, las instituciones unive $\mathrm{r}$ sitarias y los colegios profesionales ubicados en los núcleos urbanos más próximos. De la información disponible, se desprende la alta incidencia de las acciones formativas en el progreso personal y comunitario, puesto que 
en la mayoría de los aspectos consultados se alcanzan porcentajes superiores al 80\%.

A la vista de los términos en los que se han expresado todas las personas implicadas en esta iniciativa europea, así como por la alta unanimidad existente en sus valoraciones, puede afirmarse que los Cursos y Jornadas han resultado altamente positivos al contribuir al desarrollo personal, profesional y social de la población diana, así como al desarrollo endógeno del territorio donde se han aplicado.

c) Impacto: los principales protagonistas de esta formación de los recursos humanos (alumnos y docentes) se ratifican en el alto grado de contribución que supone la organización de estas actividades para el desarrollo comunitario ( $85 \%$ ). Concretan dicha contribución en aspectos tales como: facilitar conocimientos precisos para poder rentabilizar, en mayor medida, los productos propios de la zona; difundir dichos productos fuera de la comarca; elevar el nivel cultural y favorecer las relaciones sociales, encontrándose diferencias significativas a favor de los docentes y de los líderes comunitarios. A su vez, éstos subrayan que la planificación y puesta en marcha de esta iniciativa ayuda a reducir el abandono de la localidad (63\%). Reiteran que la formación constituye el elemento imprescindible y denominador común de todas las actividades encaminadas a alcanzar los objetivos que se pretendían.

A través de la realización de los diferentes cursos y jornadas técnicas se formaron a 470 personas en 16 temas diferentes, directamente vinculados a la problemática del entorno. Todos los colectivos implicados y, en especial, los líderes comunitarios y los gerentes de la entidad responsable, aseguran en un $85 \%$ de los casos, que han producido mejoras significativas en cuanto a la explotación y la calidad de los productos propios. Ha mejorado sensiblemente la situación laboral, al crearse más de 200 empleos nuevos con contrato indefinido y se han consolidado otro centenar más.
Teniendo en cuenta las referencias anteriores, podemos concluir que el Programa de Formación ha tenido un impactado muy positivo. Las personas que han participado han observado en la misma, una notable mejora en los aspectos citados. Ha supuesto un enriquecimiento de las personas y de la comarca y, en especial, en la promoción de ésta y de sus recursos.

Por otra parte, los resultados obtenidos una vez efectuado el Análisis Factorial, revelan que los campos más notables en el modelo de evaluación del Programa de Formación de la Iniciativa Comunitaria LEADER, son los siguientes, en orden de importancia concedida: metodología, motivación, interés en la participación y contribución de los acciones formativas al desarrollo de la zona.

\section{Conclusiones}

Retomando el objetivo general planteado en la investigación de evaluar la acción formativa de los Programas LEADER I y LEADER II, su adaptación, su eficacia y su impacto en la zona, podemos concluir que el Programa de formación:

u Se ha adaptado a las características de las personas y de la zona, así como a las circunstancias concurrentes.

u Ha resultado eficaz, teniendo en cuenta los logros alcanzados por los participantes, tanto en el plano personal como en el profesional. También revela dicha eficacia la creciente participación de la población en todo el proceso de aplicación de dicho Programa, traducida en la asistencia a los Cursos y en las posteriores demandas, que reflejan el interés por la continuación de las acciones formativas.

u Ha tenido un gran impacto, pues, junto a los resultados expuestos y a decir de los líderes comunitarios, existe un "antes y un después”, teniendo en cuenta la situación de refe- 
rencia o de partida, así como los logros conseguidos y la penetración social. Ha resultado ser un revulsivo. Por todo ello, se espera, con las aportaciones y demandas de todos, una mejora en su continuación orientada a solucionar los aspectos que lo precisen e incluyendo otros si fuera necesario.

A modo de síntesis final, se considera que esta investigación ha puesto en evidencia la valía de la actividad educativa del programa que nos ocupa, pudiéndose afirmar que el Programa LEADER es adecuado para la formación de los recursos humanos de la zona.

En cuanto a la contribución de este estudio, pensamos que puede ser útil para tomar las decisiones más adecuadas en futuros programas y puede, también, servir de orientación para diseñar procesos similares de evaluación en distintos contextos. En el marco de la Unión Europea, este trabajo puede constituir un modelo o pauta para evaluar los programas de formación que se llevan a cabo en las diversas zonas LEADER, siempre teniendo en cuenta la variedad de circunstancias y la diversidad de las mismas.

\section{Referencias bibliográfícas}

Ander Egg, E. (2000): Metodología y práctica del desarrollo de la comunidad. Vol. I y II. Buenos Aires-México: Lumen Humanitas.

Cernea, M. (1995): Primero la gente. Variables sociológicas en el Desarrollo Rural. México: Fondo Cultural de Economía Contemporánea.

Comisión de las Comunidades Europeas (1991): LEADER I. Comunicación 91/C 31/14 (DOC 73 de 19-3)

Comisión de las Comunidades Europeas (1992): Tratado de Maastricht. Luxemburgo:
Oficina de Publicaciones Oficiales de las Comunidades Europeas.

Comisión de las Comunidades Europeas (1993): Libro verde sobre la dimensión europea de la educación. Luxemburgo: Oficina de Publicaciones Oficiales de las Comunidades Europeas.

Comisión de las Comunidades Europeas (1994): Libro blanco. Crecimiento, competiti vidad y empleo. Retos y pistas para el siglo XXI. Luxemburgo: Oficina de Publicaciones Oficiales de las Comunidades Europeas.

Comisión de las Comunidades Europeas (1994): LEADER II. Comunicación 94/C 180/12 (DOCE 180 de 1-7)

Comisión de las Comunidades Europeas (1995): Libro blanco sobre la Educación y la Formación. Enseñar y aprender. Hacia una sociedad del Conocimiento. Luxemburgo: Oficina de Publicaciones Oficiales de las Comunidades Europeas.

Comisión de las Comunidades Europeas (2000): Memorandum sobre el aprendizaje permanente. Documento de trabajo de los servicios de la Comisión. Bruselas. Oficina de publicaciones de las Comunidades Europeas.

Comisión de las Comunidades Europeas (2001: LEADER PLUS. Decisión de las Comunidades Europeas, C (2001), 2176 de 20 de agosto.

Comisión Europea (1991): Guía de las Iniciativas Comunitarias, derivadas de la reforma de los Fondos Estructurales. $2^{\underline{a}}$ ed. Luxemburgo: Oficina de Publicaciones Oficiales de las Comunidades Europeas.

Comisión Europea (2002): Puntos de referencia europeos en Educación y Formación. Seguimiento del Consejo Europeo de Lisboa. 
Comunicación de la Comisión (COM (2002) 629.

Comisión Europea (2003): Hacia una Europa basada en el Conocimiento. La Unión Europea y la sociedad de la información. Europa en movimiento. Luxemburgo: Oficina de Publicaciones Oficiales de las Comunidades Europeas.

Marchioni, M. (2001): Comunidad y cambio social. Teoría y praxis de la acción comunitaria. Madrid: Editorial Popular.

Pascual Matesanz, R. M. (2005): Madrid: UNED (Tesis Doctoral)

Pérez Juste, R.; García Llamas, J. L.; Martínez Mediano (1995): Evaluación de Programas y Centros Educativos. Madrid: UNED.

Pérez Serrano, G. (1994): Investigación cualitativa, retos e interrogantes. Técnicas y análisis de datos. Madrid: Colección Aula Abierta. Editorial La Muralla.
Sarrate Capdevila, M. L. (1997): Educación de adultos. Evaluación de Centros y de experiencias. Madrid: Narcea.

Trilla Bernet, J. (1996): La Educación fuera de la escuela. Ámbitos no formales y educación social. $2^{\underline{a}}$ edición. Barcelona: Ariel

Ucar Martínez, J. (1997): La evaluación de la Animación Sociocultural. En Trilla Bernet, J. Animación Sociocultural. Barcelona: Ariel, págs. 194-208.

Vachon, B. (2001): El Desarrollo Local, teoría y práctica. Gijón (Asturias): Trea.

DirecCión DE la AUTORA: Rosa María Pascual Matesanz. Colegio Rural Agrupado "Ana de Austria”. Avda. de los Cortijos, nº 2. 47270 Cigales (Valladolid). Correo electrónico: rmpascual@educa.jcy.es

Fecha de recepción del artículo: 17.IX.2006

Fecha de aceptación definitiva: 2 1.XII.2006 